KYUNGPOOK Math. J. 49(2009), 801-809

\title{
Knots with a Trivial Coefficient Polynomial
}

\section{YASUYUKI MIYAZAWA}

Department of Mathematical Sciences, Yamaguchi University, Yamaguchi 7538512, Japan

e-mail : miyazawa@yamaguchi-u.ac.jp

ABSTRACT. By using a tangle decomposition of a knot, we give a method for the construction of a knot with the lowest trivial HOMFLY coefficient polynomial. Applying this, we show that there exist infinitely many 2-bridge knots with such a coefficient polynomial.

\section{Introduction}

The main subject of consideration in this paper is the HOMFLY polynomial [2], [9], [10] of a knot. First of all, we recall it. Let $L$ be an oriented link. The HOMFLY polynomial $P(L ; v, z) \in \mathbb{Z}\left[v^{ \pm 1}, z^{ \pm 1}\right]$ of $L$ is an invariant of the isotopy type of $L$, which is defined by the following formulas:

(1) $P(U ; v, z)=1$;

(2) $v^{-1} P\left(L_{+} ; v, z\right)-v P\left(L_{-} ; v, z\right)=z P\left(L_{0} ; v, z\right)$,

where $U$ is the trivial knot and $L_{+}, L_{-}, L_{0}$ are three links that are identical except near one point where they are as in Fig. 1. The second formula is called the skein relation for the HOMFLY polynomial.

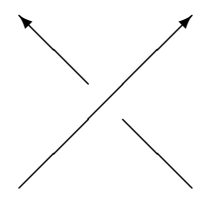

$L_{+}$

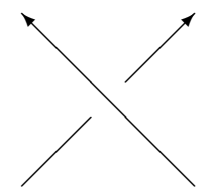

$L_{-}$

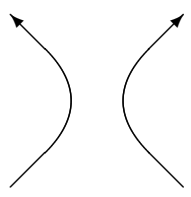

$L_{0}$

Figure 1: A skein triple

By [9], the HOMFLY polynomial of a $\mu$-component link $L$ is of the form

$$
P(L ; v, z)=\sum_{j \geq 0} P_{2 j-\mu+1}(L ; v) z^{2 j-\mu+1},
$$

Received April 5, 2009; revised July 15, 2009; accepted July 21, 2009.

2000 Mathematics Subject Classification: 57M25.

Key words and phrases: knot, HOMFLY polynomial, coefficient polynomial. 
where each Laurent polynomial $P_{2 j-\mu+1}(L ; v) \in \mathbb{Z}\left[v^{ \pm 1}\right]$ is called the $(2 j-\mu+1)$-th coefficient polynomial of $P(L ; v, z)$ in $z$ or the $(2 j-\mu+1)$-th HOMFLY coefficient polynomial. In particular, the $(1-\mu)$-th coefficient polynomial is also called the lowest coefficient polynomial. The $2 j$-th coefficient polynomial of a knot is said to be trivial if it coincides with that of the trivial knot. Furthermore, $P_{2 j-\mu+1}(L ; v) \in$ $\mathbb{Z}\left[v^{ \pm 2}\right]$ if $\mu$ is odd and $v P_{2 j-\mu+1}(L ; v) \in \mathbb{Z}\left[v^{ \pm 2}\right]$ if $\mu$ is even.

The realization problem of the HOMFLY polynomial is one of important and interesting problems in knot theory. It poses the following questions. When a given polynomial can be realized as the HOMFLY polynomial of a knot, how many knots have the polynomial as their HOMFLY polynomials? When two realizable HOMFLY polynomials are given, is there any difference between numbers of their realizing knots? If there is any difference, what does it mean? Little attention has been given to these questions. In this paper, as an approach to above questions, we are concerned with the frequency problem of the appearance of a HOMFLY coefficient polynomial. It is known that there exist non-trivial knots with the lowest trivial HOMFLY coefficient polynomial. We focus on the knot $8_{14}$ which is one of such knots. By exploring and observing tangle decomposition of $8_{14}$, we analyze the reason why such a coefficient polynomial appears and give a claim for construction of a knot with the coefficient polynomial. We also give an explicit example realizing the claim, which induces the following main result.

Theorem 1.1. There exist infinitely many 2-bridge knots with the lowest trivial HOMFLY coefficient polynomial, which have unknotting number at most two.

Remark 1.1. It is known that if a non-trivial knot has unknotting number at most two, then its $H(3)$-unknotting number is one, see [4], [3]. Thus, the 2-bridge knots given in Theorem 1.1 have $H(3)$-unknotting number one.

Remark 1.2. Kawauchi [7] has constructed an infinite family $\left\{K_{n}\right\}$ of knots having "almost" trivial HOMFLY coefficient polynomials with some additional conditions, that is, $P_{0}\left(K_{n} ; v\right)=1$ and for a given positive integer $N P_{2 k}\left(K_{n} ; v\right)=0(0<k<$ $N)$. However, these knots are not necessarily of 2-bridge.

The Kauffman polynomial [6] of a link $L, F(L ; a, z) \in \mathbb{Z}\left[a^{ \pm 1}, z^{ \pm 1}\right]$, is an invariant for a link. For a knot $K$, it is of the form

$$
F(K ; a, z)=\sum_{j \geq 0} F_{j}(K ; a) z^{j},
$$

where each Laurent polynomial $F_{j}(K ; a) \in \mathbb{Z}\left[a^{ \pm 1}\right]$ is called the $j$-th coefficient polynomial of $F(K ; a, z)$ in $z$ or the $j$-th Kauffman coefficient polynomial; See [5]. In particular, the 0-th coefficient polynomial is also called the lowest coefficient polynomial. The $j$-th coefficient polynomial of a knot is said to be trivial if it coincides with that of the trivial knot.

It is known that there is a relationship between coefficient polynomials of the HOMFLY and the Kauffman polynomials. 
Proposition 1.2([8]). For a knot $K, P_{0}(K ; v)=F_{0}\left(K ; \sqrt{-1} v^{-1}\right)$.

By Proposition 1.2, we have the following as a corollary of Theorem 1.1.

Corollary 1.3. There exist infinitely many 2-bridge knots with the lowest trivial Kauffman coefficient polynomial, which have unknotting number at most two.

\section{Tangle decomposition and coefficient polynomial}

A 2-string tangle $T$ is a pair $\left(B^{3}, t_{1} \cup t_{2}\right)$ of a 3 -ball $B^{3}$ and two properly embedded arcs $t_{1}$ and $t_{2}$ with $\partial\left(t_{1} \cup t_{2}\right)=\partial B^{3} \cap\left(t_{1} \cup t_{2}\right)$. Each endpoint of $t_{1} \cup t_{2}$ is called a endpoint of $T . T$ is called properly oriented if each $\operatorname{arc}$ of $T$ is oriented as in Fig. 2.

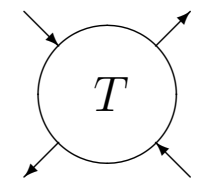

Figure 2: A properly oriented 2-string tangle

The numerator (resp. denominator) of $T$ denoted by $N(T)$ (resp. $D(T)$ ) is a link obtained from $T$ by connecting four endpoints of $T$ by two $\operatorname{arcs}$ outside $T$ as in the left (resp. right) figure of Fig. 3.
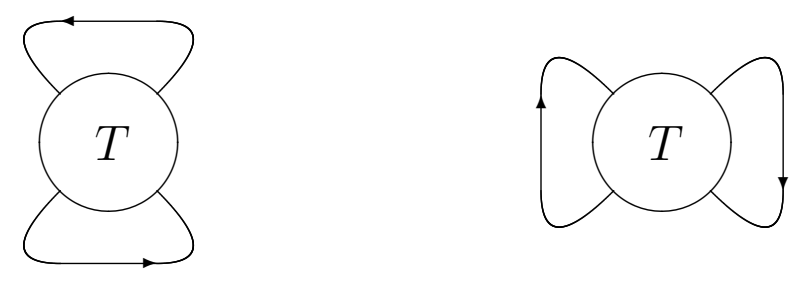

Figure 3: The numerator and the denominator

A tangle $T$ is said to be of type $N_{1}$ (resp. type $D_{1}$ ) or an $N_{1}$-tangle (resp. a $D_{1}$-tangle) if $T$ is a properly oriented 2-string tangle and $N(T)$ (resp. $D(T)$ ) is a knot.

We denote by $E_{2 n}, n \in \mathbb{Z}$, and $E_{\infty}$ tangles of type $D_{1}$ and of type $N_{1}$ as in Fig. 4, respectively. $E_{2 n}$ has $2|n|$ positive (resp. negative) crossings if $n>0$ (resp. $n<0)$ and $E_{0}$ means horizontal parallel strings without crossings.

Let $L(T), L\left(E_{0}\right)$ and $L\left(E_{\infty}\right)$ be three links identical outside a ball and inside are a properly oriented 2-string tangle $T$, the $D_{1}$-tangle $E_{0}$ and the $N_{1}$-tangle $E_{\infty}$, 


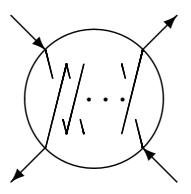

$E_{2 n}$

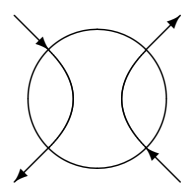

$E_{\infty}$

Figure 4: Trivial tangles

respectively.

Lemma 2.1. For a $D_{1}$-tangle $T$, there exists a unique pair $(f(T ; v, z), g(T ; v, z))$ of polynomials so that

$$
P(L(T) ; v, z)=f(T ; v, z) P\left(L\left(E_{0}\right) ; v, z\right)+v z g(T ; v, z) P\left(L\left(E_{\infty}\right) ; v, z\right),
$$

where $f(T ; v, z), g(T ; v, z) \in \mathbb{Z}\left[v^{ \pm 2}, z^{2}\right]$.

Proof. Since any properly oriented 2-string tangle can be expressed as a linear combination of $E_{0}$ and $E_{\infty}$ by linear skein theory, we easily obtain a unique pair $(f(T ; v, z), g(T ; v, z))$ of polynomials such that

$$
P(L(T) ; v, z)=f(T ; v, z) P\left(L\left(E_{0}\right) ; v, z\right)+v z g(T ; v, z) P\left(L\left(E_{\infty}\right) ; v, z\right),
$$

where $f(T ; v, z), g(T ; v, z) \in \mathbb{Z}\left[v^{ \pm 1}, z^{ \pm 1}\right]$. We only have to show that $f(T ; v, z)$ and $g(T ; v, z)$ are elements of $\mathbb{Z}\left[v^{ \pm 2}, z^{2}\right]$. Considering the HOMFLY polynomials of the numerator and the denominator of $T$, we have

$$
P(N(T) ; v, z)=f(T ; v, z) P\left(N\left(E_{0}\right) ; v, z\right)+v z g(T ; v, z) P\left(N\left(E_{\infty}\right) ; v, z\right)
$$

and

$$
P(D(T) ; v, z)=f(T ; v, z) P\left(D\left(E_{0}\right) ; v, z\right)+v z g(T ; v, z) P\left(D\left(E_{\infty}\right) ; v, z\right) .
$$

Since $D\left(E_{0}\right)$ and $N\left(E_{\infty}\right)$ are trivial knots and $N\left(E_{0}\right)$ and $D\left(E_{\infty}\right)$ are 2-component trivial links, we obtain

$$
P(N(T) ; v, z)=\left(v^{-1}-v\right) z^{-1} f(T ; v, z)+v z g(T ; v, z)
$$

and

$$
P(D(T) ; v, z)=f(T ; v, z)+\left(1-v^{2}\right) g(T ; v, z) .
$$

From these equalities, we have

$$
\left\{\left(1-v^{2}\right)-v^{2} z^{2}\right\} g(T ; v, z)=\left(1-v^{2}\right) P(D(T) ; v, z)-v z P(N(T) ; v, z) .
$$


It is easy to see that $P(D(T) ; v, z)$ and $v z P(N(T) ; v, z)$ are elements of $\mathbb{Z}\left[v^{ \pm 2}, z^{2}\right]$. It follows that $g(T ; v, z) \in \mathbb{Z}\left[v^{ \pm 2}, z^{2}\right]$, which leads to $f(T ; v, z) \in \mathbb{Z}\left[v^{ \pm 2}, z^{2}\right]$.

Remark 2.1. Since $f(T ; v, z)$ and $g(T ; v, z)$ are elements of $\mathbb{Z}\left[v^{ \pm 2}, z^{2}\right], f(T ; v, z)$ and $g(T ; v, z)$ can be expressed as $\sum_{j \geq 0} f_{2 j}(T ; v) z^{2 j}$ and $\sum_{j \geq 0} g_{2 j}(T ; v) z^{2 j}$ respectively, where $f_{2 j}(T ; v), g_{2 j}(T ; v) \in \mathbb{Z}\left[v^{ \pm 2}\right]$ for each $j$.

Lemma 2.2. For an $N_{1}$-tangle $T$, there exists a unique pair $(f(T ; v, z), g(T ; v, z))$ of polynomials so that

$$
P(L(T) ; v, z)=v z f(T ; v, z) P\left(L\left(E_{0}\right) ; v, z\right)+g(T ; v, z) P\left(L\left(E_{\infty}\right) ; v, z\right),
$$

where $f(T ; v, z), g(T ; v, z) \in \mathbb{Z}\left[v^{ \pm 2}, z^{2}\right]$.

Proof. The proof of the lemma is similar to that of Lemma 2.1.

Polynomials $f(T ; v, z)$ and $g(T ; v, z)$ which appear in Lemmas 2.1 or 2.2 are essentially determined by the tangle $T$ only. So, we call a pair $(f(T ; v, z), g(T ; v, z))$ of polynomials the normal coordinate of $T$.

Let $T$ and $S$ be properly oriented 2-string tangles. We define addition of tangles $T$ and $S$ by connecting endpoints of $T$ and $S$ as in Fig. 5 and denote it by $T+S$.

If $T$ is a $D_{1}$-tangle and $S$ is an $N_{1}$-tangle, then $T+S$ is an $N_{1}$-tangle.

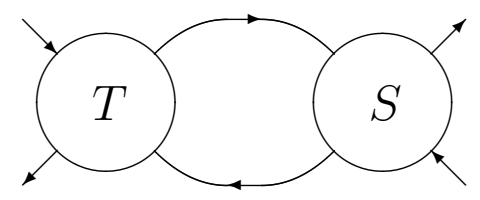

Figure 5: Addition of tangles

Lemma 2.3. Let $T$ be a $D_{1}$-tangle and $S$ an $N_{1}$-tangle. Let $(f(T ; v, z), g(T ; v, z))$ and $(f(S ; v, z), g(S ; v, z))$ be normal coordinates of $T$ and $S$, respectively. Then,

$$
\begin{aligned}
P(N(T+S) ; v, z)= & \left(1-v^{2}\right) f(T ; v, z) f(S ; v, z)+f(T ; v, z) g(S ; v, z) \\
& +v^{2} z^{2} g(T ; v, z) f(S ; v, z)+\left(1-v^{2}\right) g(T ; v, z) g(S ; v, z) .
\end{aligned}
$$

Proof. Since $N\left(E_{0}+S\right)=N(S)$ and $N\left(E_{\infty}+S\right)=D(S)$, we have

$$
\begin{aligned}
P(N(T+S) ; v, z)= & f(T ; v, z) P\left(N\left(E_{0}+S\right) ; v, z\right) \\
& +v z g(T ; v, z) P\left(N\left(E_{\infty}+S\right) ; v, z\right) \\
= & f(T ; v, z) P(N(S) ; v, z)+v z g(T ; v, z) P(D(S) ; v, z) .
\end{aligned}
$$

Since

$$
\begin{aligned}
P(N(S) ; v, z) & =v z f(S ; v, z) P\left(N\left(E_{0}\right) ; v, z\right)+g(S ; v, z) P\left(N\left(E_{\infty}\right) ; v, z\right) \\
& =\left(1-v^{2}\right) f(S ; v, z)+g(S ; v, z)
\end{aligned}
$$


and

$$
\begin{aligned}
P(D(S) ; v, z) & =v z f(S ; v, z) P\left(D\left(E_{0}\right) ; v, z\right)+g(S ; v, z) P\left(D\left(E_{\infty}\right) ; v, z\right) \\
& =v z f(S ; v, z)+\left(v^{-1}-v\right) z^{-1} g(S ; v, z)
\end{aligned}
$$

we obtain the desired formula.

The following lemma on the lowest coefficient polynomial is an immediate consequence of Lemma 2.3 and Remark 2.1.

Lemma 2.4. Under the same assumption as Lemma 2.3,

$$
\begin{aligned}
P_{0}(N(T+S) ; v)=\left(1-v^{2}\right) & f_{0}(T ; v) f_{0}(S ; v) \\
& +f_{0}(T ; v) g_{0}(S ; v)+\left(1-v^{2}\right) g_{0}(T ; v) g_{0}(S ; v) .
\end{aligned}
$$

For a tangle $T, T$ ! means the mirror image of $T$ which is the tangle obtained from $T$ by switching all crossings of $T$.

The following three lemmas are analogous to Lemmas 2.2, 2.3 and 2.4.

Lemma 2.5. Under the same assumption as Lemma 2.2,

$$
(f(T ! ; v, z), g(T ! ; v, z))=\left(-v^{-2} f\left(T ;-v^{-1}, z\right), g\left(T ;-v^{-1}, z\right)\right) .
$$

Lemma 2.6. Under the same assumption as Lemma 2.3,

$$
\begin{aligned}
P(N(T+S !) ; v, z)= & \left(1-v^{-2}\right) f(T ; v, z) f\left(S ;-v^{-1}, z\right)+f(T ; v, z) g\left(S ;-v^{-1}, z\right) \\
& -z^{2} g(T ; v, z) f\left(S ;-v^{-1}, z\right)+\left(1-v^{2}\right) g(T ; v, z) g\left(S ;-v^{-1}, z\right) .
\end{aligned}
$$

Lemma 2.7. Under the same assumption as Lemma 2.3,

$$
\begin{aligned}
P_{0}(N(T+S !) ; v)=(1 & \left.-v^{-2}\right) f_{0}(T ; v) f_{0}\left(S ;-v^{-1}\right) \\
& +f_{0}(T ; v) g_{0}\left(S ;-v^{-1}\right)+\left(1-v^{2}\right) g_{0}(T ; v) g_{0}\left(S ;-v^{-1}\right) .
\end{aligned}
$$

Proposition 2.8. Let $T$ be a tangle of type $D_{1}$ and $S$ a tangle of type $N_{1}$. Let $(f(T ; v, z), g(T ; v, z))$ and $(f(S ; v, z), g(S ; v, z))$ be normal coordinates of $T$ and $S$, respectively. Suppose that $P_{0}(N(T+S) ; v)=P_{0}\left(N(T+S) ;-v^{-1}\right)$. If $f_{0}(T ; v)=$ $f_{0}\left(T ;-v^{-1}\right)$ and $v g_{0}(T ; v)=-v^{-1} g_{0}\left(T ;-v^{-1}\right)$, then $P_{0}(N(T+S !) ; v)=P_{0}(N(T+$ $S) ; v)$.

Proof. By the assumption of the proposition and Lemmas 2.4 and 2.7, we obtain

$$
\begin{aligned}
P_{0}(N(T+S !) ; v)= & \left(1-v^{-2}\right) f_{0}\left(T ;-v^{-1}\right) f_{0}\left(S ;-v^{-1}\right)+f_{0}\left(T ;-v^{-1}\right) g_{0}\left(S ;-v^{-1}\right) \\
& +\left(1-v^{-2}\right) g_{0}\left(T ;-v^{-1}\right) g_{0}\left(S ;-v^{-1}\right) \\
= & P_{0}\left(N(T+S) ;-v^{-1}\right)=P_{0}(N(T+S) ; v) .
\end{aligned}
$$




\section{Proof of Theorem 1.1}

Let $R$ be a tangle of type $D_{1}$ such that $D(R)$ is equivalent to the trivial knot. Let $X$ and $Y$ be tangles of type $D_{1}$ and of type $N_{1}$ as in Fig. 6, respectively. We comment that $X$ comes from tangle decomposition of $8_{14}$.

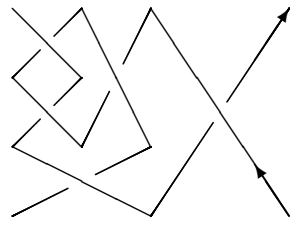

$X$

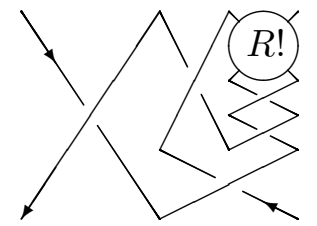

Y

Figure 6: Tangles $X$ and $Y$

The following is easily obtained by using the skein relation.

Lemma 3.1. Let $(f(X ; v, z), g(X ; v, z))$ be the normal coordinate of $X$. Then, $f(X ; v, z)=1-v^{-2} z^{2}$ and $g(X ; v, z)=\left(v^{-2}-1\right)+v^{-2} z^{2}$.

Lemma 3.2. $P_{0}(N(X+Y) ; v)=1$.

Proof. It is easy to see that $N(X+Y)$ is equivalent to $D(R !)$. Since $D(R)$ is equivalent to the trivial knot, $D(R$ !) is also equivalent to the trivial knot. Thus, we have $P_{0}(N(X+Y) ; v)=P_{0}(D(R !) ; v)=1$.

Proposition 3.3. $P_{0}(N(X+Y !) ; v)=1$.

Proof. Let $(f(X ; v, z), g(X ; v, z))$ be the normal coordinate of $X$. Then, Lemma 3.1 shows that $f_{0}(X ; v)=1$ and $g_{0}(X ; v)=v^{-2}-1$. Since $f_{0}(X ; v)=f_{0}\left(X ;-v^{-1}\right)$ and $v g_{0}(X ; v)=v^{-1}-v=-v^{-1} g_{0}\left(X ;-v^{-1}\right)$, Lemma 3.2 and Proposition 2.8 give the claim.

Any 2-bridge knot can be expressed by some continued fraction $a_{1}+\frac{1}{a_{2}+} \ldots \frac{1}{+a_{n}}$, where $a_{j} \in \mathbb{Z}, 1 \leq j \leq n$. Then, it has a diagram denoted by $C\left(a_{1}, a_{2}, \cdots, a_{n}\right)$ as in Fig. 7, where $Q$ means a 3-braid $\sigma_{1}^{-a_{1}} \sigma_{2}^{a_{2}} \cdots \sigma_{1}^{-a_{n}}$ (resp. $\sigma_{1}^{-a_{1}} \sigma_{2}^{a_{2}} \cdots \sigma_{2}^{a_{n}}$ ), which is generated by elementary 3-braids $\sigma_{1}, \sigma_{1}^{-1}, \sigma_{2}$ and $\sigma_{2}^{-1}$ as in Fig. 8, if $n$ is odd (resp. even).

Let $K_{2 n}, n \in \mathbb{Z}$, be a 2-bridge knot denoted by $C(2,1,1,2,1,1,2,2 n)$. Note that $K_{0}=C(2,1,1,2,1,1,2,0)$ is equivalent to $C(2,1,1,2,2)$ which is $8_{14}$. Since $K_{2 n}$ is a knot, the HOMFLY polynomial of $K_{2 n}$ does not depend on its orientation.

Corollary 3.4. $P_{0}\left(K_{2 n} ; v\right)=1$.

Proof. Let $X$ and $Y$ be tangles illustrated in Fig. 6. If $R=E_{2 n}$, then $N(X+Y$ !) 


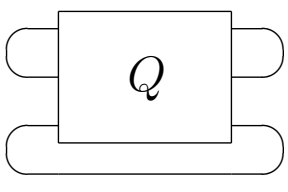

$n$ is odd

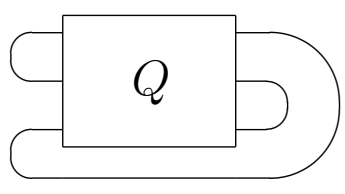

$n$ is even

Figure 7: A 2-bridge knot

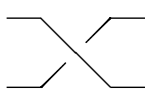

$\sigma_{1}$
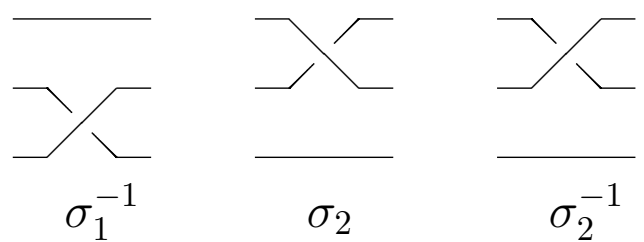

Figure 8: Elementary 3-braids

is equivalent to $K_{2 n}$. Proposition 3.3 gives the claim.

A reduced polynomial $P(L ; 1, z) \in \mathbb{Z}[z]$ obtained from the HOMFLY polynomial of a link $L$ is called the Conway polynomial [1] of $L$ and is denoted by $\nabla(L ; z)$.

Lemma 3.5. $\nabla\left(K_{2 n} ; z\right)=1-(2 n+2) z^{4}+2 n z^{6}$.

Proof. Since $K_{0}$ is equivalent to $8_{14}$, we obtain $\nabla\left(K_{0} ; z\right)=\nabla\left(8_{14} ; z\right)=1-2 z^{4}$. Let $J=C(2,1,1,2,1,1,2)$. Then, we see that $\nabla(J ; z)=2 z^{3}-2 z^{5}$. By using the skein relation of the HOMFLY polynomial at $v=1$ repeatedly, we have

$$
\nabla\left(K_{2 n} ; z\right)=\nabla\left(K_{0} ; z\right)-n z \nabla(J ; z)=1-(2 n+2) z^{4}+2 n z^{6} .
$$

The unknotting number of a knot $K$, which is denoted by $u(K)$, is the minimum number of exchanges of crossings required to deform $K$ into the trivial knot.

Lemma 3.6. $u\left(K_{2 n}\right) \leq 2$.

Proof. Let $U_{2 n}=C(2,1,-1,2,1,1,0,2 n)$. It is easy to see that $U_{2 n}$ is equivalent to the trivial knot and $K_{2 n}$ can be deformed into $U_{2 n}$ by switching two crossings. Thus, the unknotting number of $K_{2 n}$ is less than or equal to 2 .

Proof of Theorem 1.1 By corollary 3.4 and Lemmas 3.5 and 3.6, the 2-bridge knots $K_{2}, K_{4}, \cdots$ are the desired knots.

Acknowledgment. The author would like to thank the referee for helpful suggestions and comments. The author was partially supported by Grant-in-Aid for 
Scientific Research (C) (No. 20540079), Japan Society for the Promotion of Science.

\section{References}

[1] J. H. Conway, An enumeration of knots and links, in "Computational Problems in Abstract Algebra", (J. Leech, ed.), Pergamon Press, New York, 1969, pp. 329-358.

[2] P. Freyd, D. Yetter, J. Hoste, W. B. R. Lickorish, K. C. Millett and A. Ocneanu, A new polynomial invariant of knots and links, Bull. Amer. Math. Soc., 12(1985), 239-246.

[3] Y. Fujino, Y. Miyazawa and K. Nakajima, $H(n)$-unknotting number of a knot, in Proceedings of the Conference "Knots and Low Dimensional Manifolds", (1997), 72 85 .

[4] J. Hoste, Y. Nakanishi and K. Taniyama, Unknotting operations involving trivial tangles, Osaka J. Math., 27(1990), 555-566.

[5] T. Kanenobu, Kauffman polynomials as Vassiliev link invariants, in "Knots 96", (S. Suzuki, ed.), World Scientific, 1997, pp. 411-431.

[6] L. H. Kauffman, An invariant of regular isotopy, Trans. Amer. Math. Soc., 318(1990), 417-471.

[7] A. Kawauchi, Almost identical link imitations and the skein polynomial, in "Knots 90", (A. Kawauchi, ed.), Walter de Gruyter, Berlin-New York, 1992, pp. 465-476.

[8] W. B. R. Lickorish, An Introduction to Knot Theory, Graduate Texts in Mathematics, 175 Springer-Verlag, 1986.

[9] W. B. R. Lickorish and K. C. Millett, A polynomial invariant of oriented links, Topology, 26(1987), 107-141.

[10] J. H. Przytycki and P. Traczyk, Invariants of links of Conway type, Kobe J. Math., 4(1987), 115-139. 